\title{
Variation in Gastrocnemius and
}

\section{Hamstrings Muscle Activity During Peak Knee Flexor Force After Anterior Cruciate Ligament Reconstruction With Hamstring Graft: Preliminary Controlled Study}

Luna SEQUIER ${ }^{1}$, Florian FORELLI ${ }^{1}$, Maude TRAULLÉ ${ }^{1}$, Amaury VANDEBROUCK ${ }^{2}$, Pascal DUFFIET ${ }^{2}$, Louis RATTE $^{2}$,Jean MAZEAS ${ }^{1}$

${ }^{1}$ Researcher Physical Therapist, OrthoLab, 85 route de Domont, 95330 Domont, France

${ }^{2}$ Knee Orthopaedic Surgeon, Clinic of Domont, 85 route de Domont, 95330 Domont, France

\section{Abstract}

Background: The optimisation of this return to athletic activity pass by a better understanding of the behaviour of the muscle involved in knee function.

In this study, we focused on the muscular activity of the muscle involved in the flexion of the knee. Preciseley on the relation between the muscular activity of the gastrocnemius and the hamstring among the patient that underwent an anterior cruciate ligament reconstruction with hamstring graft.

Objective : The objective of the study is to compare, the muscular activity of the flexor knee muscle in patient that underwent an anterior cruciate ligament reconstruction with hamstring autograft and the individuals that have not undergone surgery.

Methods : The participants have been divided into two groups : an healthy group and an experimental group that underwent an anterior cruciate ligament recontruction with hamstring graft. The participants had to performe a strenght test on a isocinetik dynamometer. The activity of the medial gastrocnemius, lateral gastrocnemius, femoral biceps and the semitendinosus were mesured during this test.

The muscular activity of the muscle mentioned of the individuals in the first group were compared to the ones in the second group via a statistical analysis. Then, a ratio of the activity of the gastrocnemius muscle on the activity of the hamstring was calculated The results of the experimental group were then compared to the results of the control group

Results : The results showed a significative difference activity of the medial gastrocnemius, the femoral biceps and the semitendinosus muscles : the experimental group results were superior to 
the control group results However the evaluation of the activity reporting has shown significant differences in the two groups

Conclusion : This study has allowed us to show a difference in muscular activity of the gastrocnemius and hamstring muscle between patient that underwent an anterior cruciate ligament reconstruction surgery and the heathy participants. However our approch has not allowed us to identify the relation between a heihtened gastrocnemius activity and a diminished hamstring activity following an anterior cruciate ligament reconstruction with hamstring graft. Quite on the contrary, we observed a higher activity of the two muscle groups.

Nevertheless, it seem to be necessary to have a variation in situations during the analysis of the gastrocnemius muscle to fully understans its purpose in the functional activity of the knee of patient that have undergone an anterior cruciate ligament reconstruction.

Key-words : Anterior cruciate ligament; Electromyography; Isokinetic; Gastrocnemius; Hamstring

\section{Introduction}

Today the rupture of the anterior cruciate ligament $(\mathrm{ACL})$ is one of the most widespread and serious injuries in athletes, requiring in most cases a surgical repair to allow the resumption of sports practice [1]. However, despite the improvement in surgical techniques and rehabilitation procedures, only $65 \%$ of operated patients resume their sport at the same level, for the rest, $24 \%$ continue their sport at a lower level and $11 \%$ completely stop practicing sport [2]. Three parameters condition this ability to resume sport: muscle strength, neuro-motor agility, and the patient's psychological state. [3].

Independently of these parameters, the modification of the motor patterns after ACL reconstruction is a criterion that may partly explain the neuromotor disorders present in these subjects, even at the time of the resumption of sport or even in the longer term, these disorders being themselves. even identified as risk factors for future injuries.

As part of the surgery, the removal of the tendon of the semi-tendon induces a new distribution of the roles of the different flexor muscles of the knee, mainly divided into a group of hamstring muscles and a group of muscles gastrocnemius. This modification can have an important functional role, these two muscle groups not having the same function in the neuromotor control of the knee and in the protection of the ACL.

Thus, a change in the proportional activity of the flexor muscles of the knee after such a surgical intervention could lead to an alteration in the active capacities of the patients, which may result in the long term in consequences in terms of sports performance as well as risk of injury $[4,5]$.

To better understand if there is a phenomenon of modification of the activity patterns of these muscles, this study aims to compare the electromyographic activities of the hamstrings and gastrocnemius muscles during a knee flexion movement between a population operated for $\mathrm{ACL}$ reconstruction with hamstring graft and a healthy control population. Our hypothesis is that there is in the operated subjects a modification of the activity ratio between the gastrocnemius and the hamstring muscles in favor of the gastrocnemius.

\section{Materials and Methods}


This study was carried out in the format of a prospective single-center study. Study participants were not informed of the study objectives or research hypotheses. In addition, the information and data collected for the study has been systematically anonymized.

A consent form explaining the various tests performed was signed by all the participants. An authorization request has been filed with the CNIL under registration number 910191.

In total, 8 volunteer individuals participated in the study, divided into two groups of equal size: an experimental group having undergone $A C L$ reconstruction by hamstring graft, and a healthy control group. The anthropometric characteristics of the two groups evaluated have been reported in Table I below.

Table I: Descriptive table of the study population

\begin{tabular}{|c|c|c|c|c|}
\hline & Sample & Control Group & ACL Group & $P$ value \\
\hline Subject number & 8 & 4 & 4 & $\varnothing$ \\
\hline $\begin{array}{c}\text { Mean Age }( \pm S D) \text { in } \\
\text { years }\end{array}$ & $\begin{array}{c}23.75 \\
( \pm 3.58)\end{array}$ & $\begin{array}{l}23.75 \\
( \pm 0.5)\end{array}$ & $\begin{array}{c}23.75 \\
(+/-5.44)\end{array}$ & 0.76 \\
\hline Sex (Male/ Female) & $6 / 2$ & $3 / 1$ & $3 / 1$ & 0,51 \\
\hline $\begin{array}{c}\text { Mean BMI } \\
( \pm \mathrm{SD}) \text { in } \mathrm{kg} / \mathrm{m}^{2}\end{array}$ & $\begin{array}{c}23.85 \\
( \pm 1.96)\end{array}$ & $\begin{array}{c}22.9 \\
( \pm 2.23)\end{array}$ & $\begin{array}{c}24.77 \\
( \pm 1.33)\end{array}$ & 0,31 \\
\hline $\begin{array}{l}\text { Laterality (Left / } \\
\text { Right) }\end{array}$ & $4 / 4$ & $3 / 1$ & $1 / 3$ & 0,51 \\
\hline \multicolumn{5}{|c|}{ BMI: Body Mass Index |SD: Standard Deviation } \\
\hline
\end{tabular}

For the test group, one patient had been operated on for an ACL reconstruction with hamstring graft 12 weeks before the study, one patient 16 weeks before and 2 patients 24 weeks before participating in the study. For both groups, the subjects were selected according to inclusion and exclusion criteria based on the literature, making it possible to limit the biases linked to differences between the subjects as well as to standardize the study population $[4,5]$.

The control group was composed of subjects over 18 years old and with a BMI between 18.5 and 25 kg.cm - ${ }^{2}$ Subjects with a history of lower limb surgery tested within the past two years, with cardiovascular disease, central motor disease muscle damage less than 7 days, neurocognitive disorders, or having consumed alcohol or psychotropic drugs in 24 hours before participation in the study were excluded from the study protocol.

The experimental group must have been over 18 years old, have a BMI between 18.5 and 25 $\mathrm{kg} . \mathrm{cm}^{-2}$, have undergone a hamstring graft with a postoperative delay between 12 and 39 weeks before taking the measurements, have a full knee extension. Subjects with pain greater than $2 / 10$ on the numerical scale, who have undergone another surgery in the last two years on the studied limb, with iterative $A C L$ tear, with intra-articular effusion, with cardiovascular disease, presenting a central motor disease, presenting neurocognitive disorders or having consumed alcohol or psychotropic drugs within 24 hours before participation in the study were excluded from the study.

The EMG analysis is performed using a FreeEmg ${ }^{\circledR}$ capture device (BTS Bioengineering), composed of four wireless transmitters and a receiver associated with the EMGanalyzer ${ }^{\circledR}$ software, and selfadhesive foam surface electrodes, of size $30 \mathrm{~mm} \times 24 \mathrm{~mm} \mathrm{Ag} \mathrm{/} \mathrm{AgCl} \mathrm{couple.} \mathrm{All} \mathrm{data} \mathrm{is} \mathrm{collected} \mathrm{and}$ processed on a computer (VivoBook ASUS Laptop X570DD_M570DD model) it runs on Windows 10 ${ }^{\circledR}$, has a 512 GB SDD, an AMD Ryzen ${ }^{\circledR}$ processor (53500U with Radeon Vega Mobile Gfx 2100 MHz, 4 cores , 8 logical processors). 
The test protocol is based on an evaluation of the isometric and concentric strength of the various flexor muscles of the knee, performed on a HumacNorm ${ }^{\circledR}$ isokinetic dynamometer and the associated software Humac software ${ }^{\circledR}$.

The muscles studied are: Semi-Tendinosus (ST), long head of the Biceps Femoralis (BF), Medial Gastrocnemius (GM) and Lateral Gastrocnemius (GL). The placement of the electrodes on these muscles is carried out according to the European SENIAM recommendations.

An maximum voluntary isometric contraction test (MVIC) is performed for each of the muscles studied, so that these data can be used to normalize the EMG values. For more precision, 3 tests of 6 seconds are carried out for each muscle with a pause of 30 seconds between each, the best value obtained is the value retained for the normalization.

To measure flexor muscle activity, the patient is placed seated on the isokinetic dynamometer installed in the knee flexion / extension configuration. The subject's hip is at $90^{\circ}$ flexion and the knee also at $90^{\circ}$ flexion at the start of the test, the foot is left free. To correspond to certain articles in the literature, the test used is a concentric test at $90^{\circ} / \mathrm{s}$.

Once in place, the subject performs a first series of 5 movements to get used to the requested movement. The evaluation is carried out on 3 sets of 5 movements, with a 40 second break between sets. The subject is strongly encouraged during the test, aiming to focus the patient on the phase of knee flexion. All the data is collected during this test.

The processing of EMG data is recorded after acquisition and exported to an Excel spreadsheet. The data is rectified and centered first, then a moving average over 50 data is performed. Once completed, the data is normalized from the MVIC test values corresponding to each of the muscles. Finally the timestamps corresponding to the flexion phase during the tests are recorded. The average activity of each muscle during the flexion phase is thus calculated, and a ratio of Hamstring / Gastrocnemius activity is calculated.

A statistical analysis is then carried out, using the $\mathrm{R}{ }^{\circledR}$ software. The confidence interval is set such that $\alpha=0.05$, so any test with a $p$-value less than 0.05 is considered statistically significant in this study. For some anthropometric data (age, BMI) the mean is used to characterize the data, with a standard deviation as a measure of dispersion. For these same quantitative variables, a Wilcoxon test was applied in order to compare the homogeneity of the two populations (Table I). For the qualitative variables such as the sex or the laterality of the measurement, the Chi2 test was carried out in order to compare the homogeneity of the populations (Table I).

\section{Results}

The muscle activity ratios of the two groups have been reported in Figure 1 below. Statistical analysis reveals significant results concerning the mean muscle activity of $B F\left(p=5,394.10{ }^{-6}\right), G M(p=$ $0.004901)$ and ST $\left(p=1,822.10^{-6}\right)$. However, the analysis of the mean GL muscle activity does not show significant links between the two groups $(p=0.2322)$.

\begin{tabular}{|c|c|c|c|}
\hline Muscle & Control Group & ACL Group & P value \\
\hline Gastrocnemius Medialis & $\mathbf{0 , 7 2 6}$ & $\mathbf{1 , 0 5 6}$ & 0,004901 \\
\hline Gastrocnemius Lateralis & $\mathbf{0 , 6 4 9}$ & $\mathbf{0 , 5 7 1}$ & 0,2322 \\
\hline Biceps Femoris & $\mathbf{0 , 5 1 1}$ & $\mathbf{0 , 6 0 4}$ & $5,394.10^{-6}$ \\
\hline
\end{tabular}




\begin{tabular}{|c|c|c|c|}
\hline Semi-Tendinosus & $\mathbf{0 , 4 8 8}$ & $\mathbf{0 , 6 2 3}$ & $1,822.10^{-6}$ \\
\hline $\begin{array}{c}\text { Gastrocnemius/Hamstring } \\
\text { Ratio } \\
\text { ( } \pm \text { Standard Deviation) }\end{array}$ & $1,341( \pm 0,497)$ & $1,366( \pm 0,859)$ & 0.4751 \\
\hline
\end{tabular}

Figure 1: Gastrocnemius and Hamstring muscle activity ratio

\section{Discussion}

The rate of iterative $A C L$ rupture in operated patients is $5-6 \%[6]$, however this rate is offset by the fact that only $53 \%$ of patients regain their pre-injury performance level [7] . It therefore seems relevant to look at the parameters that determine the return to initial sporting activity. The 3 main parameters considered are: psychological parameters, strength and neuromotor agility.

In this study we focused on the relationship between muscle activity and gastrocnemius and hamstring in people who had surgery for reconstruction of the anterior cruciate ligament with hamstring graft. We thus analyzed the muscle activity of gastrocnemius and hamstrings during knee flexion via EMG analysis. The measurements were taken during a force test performed on an isokinetic dynamometer.

The objective of this study was to determine if there is a difference in muscle activity in the gastrocnemius and hamstring muscles in a group that underwent ACL reconstruction compared to a control group that did not undergo surgery. Before this study, several authors were also interested in this subject:

Tagesson et al [4] studied the muscle activity of the knee muscles during functional tests such as walking, open chain knee extension, uni and bipodal squats. They compare the muscle activity of the side that has undergone $A C L$ reconstruction in comparison with the healthy side. The post-surgical period considered is 5 weeks. They are therefore interested in a postoperative phase that is more immediate than that considered in our study, which is at least 12 weeks. Participants in this study performed several exercises during which muscle activity was measured via EMG and anterior tibial translation.

They observed in particular that during walking, the extension of the knee in an open chain, the squat on one leg, the squat in bipodal, the average activity of the hamstrings is higher for the limb having undergone an ACL reconstruction. They also note that the activity of the gastrocnemius is more important for the action of standing on one leg or in a bipodal squat. Thus, their results tend to show that beyond an increase in the activity of hamstrings in the operated limb, the gastrocnemius plays an agonist role to that of hamstring muscles. However, they temper these results by saying that higher muscle activity for hamstrings may be the result of the inflammatory reaction still operating at the level of the donor site 5 weeks after surgery.

Alkjær et al in 2020 [5] performed an EMG analysis of the different muscles of the knee in patients who had undergone $A C L$ reconstruction during a front knee lunge. This study reports a significant increase in medial gastrocnemius activity during a front cleft in subjects operated on 10 months ago for $\mathrm{ACL}$ reconstructive surgery compared to a healthy control group. On the other hand, the same study demonstrates that the activity of BF and ST are comparable in patients having undergone $\mathrm{ACL}$ reconstruction compared to the control group. 
The authors conclude that there is no difference in muscle activity, only that the speed of movement is affected in patients who have undergone ACL reconstruction.

Madhavan and Shields [8] study the activity of the quadriceps and Jls in a group having undergone $\mathrm{ACL}$ reconstruction about 4 years before the study in comparison with a healthy control group and also highlight $\mathrm{JI}$ activity. higher for the operated group than for the control group. However, the postoperative delays considered in Madhavan and Shields are much longer than for the present study, which limits the analogy between the two works.

All of these studies $[4,5,9]$ focus on the observation of muscle activity in the muscles involved in knee function during functional tests that solicit the neuromotor agility of participants. The present study makes it possible to show that, the increase in the activity of hamstring muscles on the one hand and of the medial gastrocnemius on the other hand, is also present during a pure force test.

Morgan et al [9] analyze the muscular activity of the BF and ST, quadriceps (vastus medial and vastus lateralis) and gastrocnemius muscles during a unipodal jump landing ("Drop jump test ") in healthy subjects. Their results show that the hamstrings and the gastrocnemius work synergistically as stabilizers of the lower limb and therefore as protectors of the ACL during this phase of reception of the jumps. Morgan et al also highlight the role of the gastrocnemius as a support for the lower limb. They therefore recommend the development of programs aimed at increasing the strength of gastrocnemius in preventing $\mathrm{ACL}$ rupture.

Another study carried out by Damavandi et al [10] focused on EMG analysis of the muscles of the knee during multidirectional disturbances in healthy subjects. They observe muscle activity of the quadriceps (vastus lateralis and vastus medialis), hamstrings (BF and ST) and gastrocnemius (medial and lateral) in two situations: the knee in extension and the knee in $30^{\circ}$ flexion. This study tends to show that the gastrocnemius are the main players in the stabilization of the knee in extension.

The studies by Damavandi and Morgan highlight the major role of the gastrocnemius as stabilizers of the lower limb, thus giving these muscles a protective function of the ACL.

However, such an interpretation should be qualified. Indeed, these studies are in disagreement with the model of Adouni [11] which studies, via the use of a biomechanical model, the effect of the modification of the activity of the gastrocnemius muscles on the active and passive responses of. knee alteration, during walking and during knee flexion from 0 to $90^{\circ}$.

Its analysis takes into account an isolated action of the gastrocnemius on the femoro-tibial joint during flexion. His study shows that, under an isolated action of the gastrocnemius muscles, the stresses present in the ACL increase. He also observed that the stresses induced by isolated activation of the gastrocnemius muscle increased with the angle of knee flexion.

Adouni's findings suggest that gastrocnemius and hamstrings although both knee flexors may act antagonistically to ACL. That is to say that the hamstrings would act in a protective manner by reducing the stresses present in the $A C L$ while the gastrocnemius on the contrary, increase the stresses present in the ACL. Thus, although present in the stabilizing reactions of the lower limb, the action of gastrocnemius would induce additional stresses on the ACL. Taking into account Adouni's observations, an increase in the activity of the gastrocnemius muscles increases the risk of $A C L$ rupture.

However, Adouni also underlines the limits of his biomechanical model of the knee joint complex. In fact, this does not take into account muscle co -contractions but only considers the isolated action of the muscles on the femoro-tibial joint.

In this context, our study, which highlights an increase in GM activity during a strength test in patients who have undergone a surgery with hamstring graft, underlines that special attention must be paid to the gastrocnemius muscles during rehabilitation of $A C L$ repair. Indeed, a modification of 
the motor pattern as a consequence of this intervention, and in particular an overuse of the gastrocnemius muscles, can prove to be deleterious. According to Adouni's model, an increase in gastrocnemius activity could increase the risk of rupture of the anterior cruciate ligament.

On the other hand, in general, overuse of muscles can cause muscle fatigue. However, muscle fatigue has been shown to increase the risk of injury [12] .

The modification of the reciprocal activity of the flexor muscles of the knee found in our study, mainly the increase in the activity of the medial gastrocnemius, can therefore lead us to the hypothesis that there is, in subjects operated on for reconstruction $A C L$ with hamstring graft, an alteration in motor patterns that can lead to both a performance deficit when resuming sports activities, but also a greater risk of injury due to the imbalance of muscle forces applying to the knee.

\section{Limits}

The present study has different limitations in terms of method and protocol used. These limits can constitute avenues for reflection in order to improve the study protocol and thus the relevance of the results.

First, the size of the study sample $(n=8)$ is small and therefore does not allow conclusions to be drawn applicable to the general population operated on for the anterior cruciate ligament. A larger sample could have allowed us to stratify the experimental group according to the postoperative delay, which would have added precision to our observations. Indeed, the postoperative delay for the participants in the experimental group is variable: between 12 and 24 weeks. We could, for example, imagine that the differences between the healthy group and the experimental group become blurred as the time elapsed since the operation or on the contrary that the motor pattern is found to be permanently modified following a reconstruction of the ACL.

On the other hand, some authors use functional tests $[4,5,9]$ which could be more relevant for the normalization of gastrocnemius. Another method considered may be to normalize the signal directly using as a reference value the peak of muscle activity during the test exercise $[10,13]$. These tests were not used in the present study because their reproducibility is less reliable than for a maximal isometric contraction and this does not follow the recommendations of SENIAM. In addition, we know that muscle activity measured by EMG is greater in a dynamic contraction mode than in an isometric contraction mode [13] . This allows us to say that for this type of measurement it would be relevant to carry out the normalization with respect to a maximum voluntary contraction in a dynamic and non-isometric contraction mode.

However, these modifications do not modify the results that we were able to find in this study, but simply the conclusions that we can deduct from them.

\section{Conclusion}

The objective of our work was to compare the muscle activity of the gastrocnemius and hamstring muscles in patients having undergone surgery after $\mathrm{ACL}$ reconstruction with a healthy control group.

This study highlights the fact that, in subjects who underwent ACL reconstruction with hamstring graft, there is greater activity of the hamstring muscles as well as the medial gastrocnemius than in a healthy population.

On the other hand, our approach could not determine a difference in relative activity between the gastrocnemius muscles and the hamstring muscles. 
By taking into account the activity of gastrocnemius in our muscle evaluation, this study highlights their key role in post-surgery rehabilitation in comparison with more classic approaches focused on the recovery of muscle strength only of the quadriceps and hamstrings. Indeed, an alteration of the motor pattern inducing an overuse of the gastrocnemius could cause secondary injuries or even an iterative rupture of the ACL. It is therefore necessary to take this alteration into account in the motor pattern of patients who have been operated on for reconstruction of the ACL reconstruction with hamstring graft, in order to optimize the return to a sporting activity.

However, an analysis of the muscle activity of gastrocnemius in more varied situations, especially in sports activities, seems necessary for a better understanding of the role of this muscle group in the functional activity of the knee in patients who have undergone $A C L$ reconstruction.

\section{Bibliography}

1. Kaeding CC, Léger-St-Jean B, Magnussen RA. Epidemiology and Diagnosis of Anterior Cruciate Ligament Injuries. Clin Sports Med. Jan 2017; 36 (1): 1 - 8.

2. Crawford SN, Waterman MBR, Lubowitz JH. Long-Term Failure of Anterior Cruciate Ligament Reconstruction. Arthrosc J Arthrosc Relat Surg. 2013 Sep 1; 29 (9): 1566 - 71.

3. Ardern CL, Glasgow P, Schneiders A, Witvrouw E, Clarsen B, Cools A, et al. 2016 Consensus statement on return to sport from the First World Congress in Sports Physical Therapy, Bern. Br J Sports Med. 2016 Jul ; 50 (14): 853 - 64.

4. Tagesson S, Oberg B, Kvist J. Tibial translation and muscle activation during rehabilitation exercises 5 weeks after anterior cruciate ligament reconstruction. Scand J Med Sci Sports. 2010 Feb ; 20 (1): $154-64$.

5. Alkjær T, Smale KB, Flaxman TE, Marker IF, Simonsen EB, Benoit DanielL, et al. Forward lunge before and after anterior cruciate ligament reconstruction: Faster movement but unchanged knee joint biomechanics. Williams JL, editor. PLOS ONE. Jan 24, 2020; 15 (1): e0228071.

6. Crawford SN, Waterman MBR, Lubowitz JH. Long-Term Failure of Anterior Cruciate Ligament Reconstruction. Arthrosc J Arthrosc Relat Surg. 2013 Sep 1; 29 (9): 1566 - 71.

7. Ardern CL, Webster KE, Taylor NF, Feller JA. Return to the Preinjury Level of Competitive Sport After Anterior Cruciate Ligament Reconstruction Surgery: Two-thirds of Patients Have Not Returned by 12 Months After Surgery. Am J Sports Med. 2011 Mar; 39 (3): 538 - 43.

8. Madhavan S, Shields RK. Neuromuscular responses in individuals with anterior cruciate ligament repair. Clin Neurophysiol Off J Int Fed Clin Neurophysiol . 2011 May ; 122 (5): 997 - 1004.

9. Morgan KD, Donnelly CJ, Reinbolt JA. Elevated gastrocnemius forces compensate for decreased hamstrings forces during the weight-acceptance phase of single-leg jump landing: implications for anterior cruciate ligament injury risk. J Biomech . 2014 Oct; 47 (13): 3295 - 302.

10. Damavandi M, Mahendrarajah L, Dixon PC, DeMont R. Knee joint kinematics and neuromuscular responses in female athletes during and after multi-directional disturbances. Hum Mov Sci. Apr 2020; 70: 102596 .

11. Adouni M, Shirazi- Adl A, Marouane H. Role of gastrocnemius activation in knee joint biomechanics: gastrocnemius acts as an $\mathrm{ACL}$ antagonist. Comput Methods Biomech Biomed Engin . 2016; 19 (4): 376 - 85. 
12. Jones CM, Griffiths PC, Mellalieu SD. Training Load and Fatigue Marker Associations with Injury and IIIness: A Systematic Review of Longitudinal Studies. Sports Med. May 2017; 47 (5):

$943-74$.

13. Ball N, Scurr JC. Efficacy of current and novel electromyographic normalization methods for lower limb high-speed muscle actions. Eur J Sport Sci. 2011 Nov ; 11 (6): 447 - 56. 